\title{
Effect of Orthognathic Surgery for Correction of Class III Facial Deformities in the Development of Obstructive Sleep Apnea
}

\author{
Caio Cesar de Souza Loureiro, ${ }^{1}$ Astrid Virginia Buysse Temprano, ${ }^{2}$ and \\ Luiz Fernando Lobo Leandro ${ }^{2}$ \\ ${ }^{1}$ Brazilian Society of Oral \& Maxillofacial Surgery, Sobracibu, Brazil \\ ${ }^{2}$ Oral and Maxillofacial Surgery Service of the Hospital Santa Paula, São Paulo, Brazil
}

Correspondence to :

Caio Cesar de Souza Loureiro

E-mail : csloureiro@gmail.com

Keywords :

sleep apnea, obstructive, oral sur-

gery, prognathism

\begin{abstract}
Obstructive sleep apnea is recognized as a major respiratory disorder in adults, characterized by a sharp decline or periodic interruption of airflow during sleep. The exact cause of this condition is uncertain and apparently multifactorial. Prognathic (Class III) cases are generally subject to the procedures of mandibular retrusion through sagittal ramus osteotomies that change the characteristics of the oropharynx, causing reduction of the airway space. The aim of this paper is to review the literature on the influence of orthognathic surgery for the correction of prognathism in the development of postoperative respiratory disorders such as obstructive sleep apnea.
\end{abstract}

\section{Introduction}

Obstructive sleep apnea (OSA) is recognized as a major respiratory disorder in adults (1). The exact cause of this condition is uncertain and apparently multifactorial (2). Obesity, sex, age, and race appear to be predisposing factors for the development of OSA (3).

Patients with this disorder experience sleep deprivation due to numerous episodes of sleep disruption during the night and the inability to reach the stage of rapid eye movement sleep, which causes daytime sleepiness in approximately $90 \%$ of those affected (4), increasing susceptibility to physiological changes and the risk of medical complications as a result of nocturnal hypoxia and hypercapnia $(5,6)$.

Orthognathic surgery is increasingly used for the correction of facial deformities (congenital or acquired) to restore the functions of the stomatognathic system. The surgery for maxillomandibular advancement is now one of the most effective procedures for the treatment of OSA, due to the expansion of the retro-palatal and retro-lingual airways, with a cure rate of over $90 \%(7,8)$.

Moreover, prognathic (Class III) cases are gener- ally subject to mandibular retrusion through sagittal ramus osteotomies (9), which shift the oropharynx backwards, causing reduction of the airways $(10,11)$.

The aim of this paper is to review the literature on the influence of orthognathic surgery for the correction of prognathism in the development of postoperative respiratory disorders such as OSA.

\section{Literature Review}

The surgical procedure for mandibular retrusion through sagittal ramus osteotomies is often performed for the correction of prognathism (9), causing changes in the morphology of the lower face, pharynx, and upper airspace (11-14). These changes may occur at different levels such as the oropharynx, nasopharynx, and hypopharynx $(15,16)$.

This is mainly due to morphological changes and adaptation of anatomical structures related to movement of the jaw after surgery, especially those of the tongue and hyoid bone along with their associated muscles $(17,18)$.

According to several studies, the retro-mandibular positioning results in the posterior and inferior movements of the hyoid bone in the postoperative 
period, causing relaxation of the supra-hyoid muscles and pulling the tongue in the same direction by changing its position in the reduced oral cavity $(9,15$, 17). The subsequent placement of the tongue, along with its relationship to the soft palate and muscle relaxation, can cause changes in the oropharyngeal morphology, decreasing the oropharyngeal airspace and increasing the respiratory resistance. This leads to problems, such as OSA $(11,12,14)$, which may be more severe when other predisposing factors such as enlarged adenoids or other bone changes, such as posterior vertical maxillary deficiency, are present (13).

The exact correlation between mandibular retrusion and the development of respiratory disorders is not fully understood due to the contradictory findings of various studies.

Although a decreased airway after surgery is a common finding, the relationship between respiratory resistance and decreased oropharyngeal space over time remains contradictory (11).

Some studies have suggested that changes in the oropharyngeal complex caused by the retraction of the mandible are only temporary phenomena observed over a relatively short time during the postoperative period (9). After they are observed, the hyoid bone could return to its original position in theory depending on the size of the pharynx $(9,10,17)$.

However, other studies show that the movement of the hyoid bone back to its original position does not influence the increase of the reduced airways $(15,17)$. In contrast, superior repositioning of the tongue through the anterior and superior movements of the hyoid bone tends to increase its contact with the soft palate and may increase the reduction of the retropalatal space (9).

As compensation, there is a tendency to an anterior hyperextension of the head, easily detected on lateral radiographs taken in the postoperative period ; this head position increases the permeability of the upper airway and masks morphological changes (17).

Although cephalometric radiography represents a two-dimensional view for evaluating a three-dimen- sional problem, a good correlation has been found between the size of the airways shown in lateral radiographs and those shown in three-dimensional computed tomography scans, providing useful information for assessing the anatomic relationships of the airway structures and estimating the volumes of the nasopharynx and tongue $(13,14)$.

Thus, cephalometric radiography is valuable in planning surgical procedures for patients with Class III cases, because of the importance of clarifying the morphological relationship between the lower third of the face and the airway $(11,14)$.

Independently of the hyoid bone and tongue anatomical variations, a positive correlation has been found between the degree of mandibular retrusion and changes in the pharyngeal area, although the exact relationship has not been defined $(10,12,14)$.

Thus, knowledge of the minimum critical size of the airways is of great importance for surgical planning, in an attempt to predict the reduction of airspace during the postoperative period, and thus avoid the risk of developing respiratory disorders related to sleep $(14,16)$. It is important to remember that changes in mandibular skeletal dimensions are assessed and handled during the predictive study only in the sagittal axis, while changes in pharyngeal soft tissues should be considered with a three-dimensional perspective (12).

The estimated value of the pharyngeal airspace, which is obtained by measuring the narrowest dimension between the base of the tongue or soft palate and the posterior pharyngeal wall in a cephalometric radiograph, is 10 to $12 \mathrm{~mm}(12-14)$.

Therefore, we can say that in the absence of other anatomical changes, small posterior jaw movements do not exert significant changes in the permeability of the airways. Conversely, in cases of major discrepancies, where the movement is more than $10 \mathrm{~mm}$, the retrusion of the mandible can cause a significant reduction in the pharynx and the development of OSA $(12,15)$. In these cases, maxillary advancement surgery or a combination of maxillary advancement and mandibular retrusion alternatives should be considered in surgical planning (12). 


\section{Discussion}

Obstructive sleep apnea is a serious disorder characterized by a sharp decline or even the periodic interruption of the airflow of an individual during sleep; its prevalence varies from $1.3 \%$ to $4 \%$ in middle-aged male patients (1).

As a result of the deprivation of rest following several episodes of sleep disruption, $90 \%$ of the patients with this condition experience daytime sleepiness, which can lead to cognitive changes affecting productivity and behavioral changes, such as memory loss, difficulty concentrating, irritability, depression, and even decreased libido or impotence (19).

Furthermore, the episodes of breathing interruption may cause hypoxia and hypercapnia, which can lead to the development of serious diseases such as arrhythmias, ischemic heart disease, and hypertension, pulmonary or neurological complications, and even death (5).

The causes for the development of OSA are not yet fully known, but apparently its etiology is multifactorial (2). Several studies point to obesity, male sex, age, ethnicity, and craniofacial morphology as predisposing factors for developing this disorder (3). Anatomical features such as hyperplasia of the adenoids, macroglossia, narrow nostrils, nasal turbinate hypertrophy, septal deviation, elongated soft palate, and airway tumors also appear to have a significant role in the pathogenesis of OSA $(13,20$, 21).

Moreover, patients with OSA usually present common clinical and radiographic characteristics such as mandibular retrognathia, maxillary retrusion, posterior vertical deficiency of the maxilla, retro-positioning of the tongue, high occlusal and mandibular planes, reduction in pharyngeal space on cephalometric radiographies, poorly defined gonial angles, and Class II occlusion (13).

Orthognathic surgery, through maxillo-mandibular advancement, was introduced as an alternative to tracheostomy for the treatment of OSA in 1979 and is currently one of the most effective methods for managing this disorder, with cure rates ranging from $90 \%$ to $100 \%(7,8)$.

The coordination of the advancement of the mandible and maxilla causes stretching of the soft tissues of the oropharynx, resulting in expansion of the retro -palatal and retro-lingual airways. However, the success of this procedure seems to be associated with large skeletal movements, generally greater than 10 $\mathrm{mm}(8,22)$.

If the surgically advanced maxillo-mandibular complex is capable of promoting increased permeability of the airway, attention must be given to Class III cases in patients about to undergo orthognathic surgery for the correction of a facial deformity.

Patients with prognathism commonly undergo surgical procedures for mandibular retrusion that may result in a reduced volume of pharyngeal airspace $(9$, 11-14).

Numerous studies have shown that significant morphological changes occur after mandibular retrusion, especially with respect to the tongue and hyoid bone and their associated muscles. In these cases, the posterior and inferior movements of the hyoid bone are a common finding, with the suprahyoid muscles pulling in the same direction, causing retro-positioning of the tongue $(9,15,17,18)$.

The new posture of the tongue and its relationship with the tissues of the soft palate tend to reduce the retro-palatal airspace in a way similar to that of relaxation of the supra-hyoid muscles, which could mean a reduction of retro-pharyngeal space $(11,12$, 14).

Although in some cases this change is considered temporary, because there is a postoperative tendency of the hyoid bone to return to its original position, this is not reflected in the recovery of respiratory volume $(15,17)$.

Long-term studies show that even after postoperative periods of at least 15 years, restoration the retro-pharyngeal space has not occurred $(12,14)$.

However, the relationship between the movement of soft tissue and the lower airway are poorly understood (11).

The consensus in the literature is that there is a direct relationship between the decrease in the vol- 
ume of the airways and the degree of movement of mandibular retrusion $(10,12,14)$. Given that the normal pharyngeal airspace measured on cephalometric radiographs averages 10 to $12 \mathrm{~mm}$, a major change to the airways as a result of small posterior movements of the jaw should not be expected (12-15). However, large mandible retrusion (10 $\mathrm{mm}$ or more) should be evaluated with criteria and should be considered during the surgical planning of maxillary advancement with mandibular retrusion (12).

\section{Conclusion}

Orthognathic surgery for mandibular retrusion is commonly performed to correct maxillo-mandibular prognathism.

This procedure causes morphological and structural changes in soft tissues, leading to a decrease in the volume of the airways, which may result in the development of respiratory disorders such as obstructive sleep apnea.

Proper surgical planning is essential to minimize the risks of surgical induction of these changes. Implementation of a combined procedure of mandibular retrusion and maxillary advancement is recommended when the skeletal discrepancy exceeds $10 \mathrm{~mm}$.

Particular attention should be given to patients with other anatomical changes, such as hyperplasia of the adenoids, enlarged tongue, and elongated soft palate, and to male patients, obese patients, and elderly patients.

\section{References}

1. Young T, Palta M, Dempsey J: The occurrence of sleep-disordered breathing among middle-aged adults. New Engl J Med, 328 : 1230, 1993.

2. Cherniack NS: Respiratory dysrhythmias during sleep. N Engl J Med, 305 : 325-330, 1981.

3. Li KK, Riley RW, Powell NB, Troell R, Guilleminault C : Overview of phase II surgery for obstructive sleep apnea syndrome. Ear Nose Throat J, 78: 851-857, 1997.

4. Lye KW, Waite PD, Meara D, Wang D : Quality of life evaluation of maxillomandibular advancement surgery for treatment of obstructive sleep apnea. J Oral
Maxillofac Surg, 66: 968-970, 2008.

5. Peppard PE, Young T, Palta M : Prospective study of the association between sleep-disordered breathing and hypertension. N Engl J Med, 342 : 1378-1382, 2000.

6. Peker Y, Hedner J, Norum J : Increased incidence of cardiovascular disease in middle-aged men with obstructive sleep apnea: A 7-year follow-up. Am J Respir Crit Care Med, 166 : 159-165, 2002.

7. Li KK, Guilleminault C, Riley RW : Obstructive sleep apnea and maxillomandibular advancement: An assessment of airway changes using radiographic and nasopharyngoscopic examinations. J Oral Maxillofac Surg, 60: 526-530, 2002.

8. Fairburn SC, Waite PD, Vilos G: Three-dimensional changes in upper airways of patients with obstructive sleep apnea following maxillomandibular advancement. J Oral Maxillofac Surg, 65 : 6-12, 2007.

9. Eggensperger N, Smolka W, Iizuka T: Long-term changes of hyoid bone position and pharyngeal airway size following mandibular setback by sagittal split ramus osteotomy. J Cranio-Maxillofac Surg, 33 : 111117, 2005.

10. Tselnik M, Pogrel MA: Assessment of the pharyngeal airway space after mandibular setback surgery. J Oral Maxillofac Surg, 58 : 282-285, 2000.

11. Saitoh K: Long-term changes in pharyngeal airway morphology after mandibular setback surgery. Am J Orthod Dentofacial Orthop, 125 : 556-561, 2004.

12. Hochban W, Schurmann R, Brandenburg U, Conradt $\mathrm{R}$ : Mandibular setback for surgical correction of mandibular hyperplasia-does it provoke sleep disorder? Int J Oral Maxillofac Surg, 25 : 333-338, 1996.

13. Mehra P, Downie M, Pita MC, Wolford LM : Pharyngeal airway space changes after counterclockwise rotation of the maxillomandibular complex. Am J Orthod Dentofacial Orthop, 120 : 154-159, 2001.

14. Liukkonen M, Vahatalo K, Peltomaki T, Tiekso J, Happonen RP : Effect of mandibular setback surgery on the posterior airway size. Int J Adult Orthod Orthognath Surg, 17: 41-46, 2002.

15. Samman N, Tang SS, Xia J : Cephalometric study of the upper airway in surgically corrected Class III skeletal deformity. Int J Adult Orthod Orthognath Surg, 17 : 180-190, 2002.

16. Chen F, Terada K, Hanada K, Saito I : Predicting the pharyngeal airway space after mandibular setback surgery. J Oral Maxillofac Surg, 63 : 1509-1514, 2005.

17. Kawakami M, Yamamoto K, Fujimoto M, Ohgi K, Inoue $\mathrm{M}$, Kirito $\mathrm{T}$ : Changes in tongue and hyoid positions and posterior airway space following mandibular setback surgery. J Cranio-Maxillofac Surg, $33:$ 107-110, 2005.

18. Chen F, Terada K, Hua Y, Saito I : Effects of bimaxillary surgery and mandibular setback surgery on phar- 
yngeal airway measurements in patients with Class III skeletal deformities. Am J Orthod Dentofacial Orthop, 131: 372-377, 2007.

19. Parish JM, Lyng PJ : Quality of life in bed partners of patients with obstructive sleep apnea or hypopnea after treatment with continuous positive airway pressure. Chest, 124 : 942-948, 2003.

20. Lowe AA, Gionhaku N, Takeuchi K, Fleetham JA: Three-dimensional $\mathrm{CT}$ reconstructions of tongue and airway in adult subjects with obstructive sleep apnea. Am J Orthod Dentofacial Orthop, 90 : 364-374, 1986.

21. Palmer LJ, Redline S: Genomic approaches to understanding obstructive sleep apnea. Respir Physiol Neurobiol, 135 : 187-205, 2003.

22. Hochban W, Brandenburg U, Peter JH : Surgical treatment of obstructive sleep apnea by maxillomandibular advancement. Sleep, 17 : 624-628, 1994. 